\title{
Molecular modeling of a predominant $\beta$-CGTase G1 and analysis of ionic interaction in CGTase
}

\begin{abstract}
The protein 3D structure for CGTase G1 was determined by homology modeling and a good structure was generated after three rounds of energy minimization process. The ERRAT and Verify3D scores for the predicted structure were determined as 98.07 and $90.991 \%$, respectively. The presences of ionic interactions inside the CGTase G1 structure were compared with five CGTases crystal-structures. Mesophilic CGTases have lesser numbers of ionic pairs (average of 72.3 pairs) than of the thermophilic CGTases (average of 78.6 pairs). Most of the interactions in CGTases were involved in the form of networking. The average number for networking ionic pairs in thermostable and mesophilic CGTases is 69.3 and 62.7, respectively. Most of the ionic interactions in CGTases were found in Domain A and the most complex ionic networking was located in this catalytic domain as well. These chargedresidues generate interlinking networking that covers a huge area that surrounds the active site groove. A few numbers of secondary structures strands were interlinked by the ionic interactions and this creates a natural protection for the catalytic TIM-barrel structure (Domain A) against heat. Most of the residues involved are consensus, however, slight variations were found. The triad Asp181-Arg185-Asp175 might plays an important factor in the networking which causes the half life of CGTase G1 to be slightly higher compared to other CGTases originally produced by mesophilic strains.
\end{abstract}

Keyword: Cyclodextrin glucanotrans ferase; Homology modeling; Ionic interaction; Protein salt bridge; Protein stability 